\title{
Resultados Clínicos de Pacientes com Reestenose Intrastent Não Tratada com Novo Procedimento de Revascularização
}

\author{
Carlos Antonio Mascia Gottschall'1, Rogério Sarmento-Leite', \\ Dulce Welter ${ }^{1}$, Alexandre Schaan de Quadros ${ }^{1}$
}

\section{RESUMO}

Introdução: A reestenose clínica após o implante de stent coronário costuma ser tratada com revascularização da lesão-alvo (RLA). Nosso objetivo foi relatar o perfil clínico e os desfechos de pacientes com reestenose intrastent (RIS) sintomática que não foram submetidos a nova RLA. Método: Identificamos pacientes que apresentaram reestenose clínica após colocação de stent coronário entre janeiro de 1997 e dezembro de 2001. As características clínicas e angiográficas e os desfechos clínicos dos pacientes que não passaram por nova revascularização (grupo sem RLA) foram comparados aos de pacientes revascularizados (grupo com RLA). Todos os pacientes tiveram acompanhamento de pelo menos dois anos após implante do stent para ocorrência de eventos cardíacos adversos maiores (ECAM). Resultados: No período do estudo, 1.221 stents foram implantados em 1.149 pacientes. Observou-se RIS em 135 pacientes (12\%), dos quais 104 tiveram acompanhamento clínico e angiográfico completo, 23 no grupo sem RLA e 81 no grupo com RLA. O período médio de acompanhamento após a colocação de stent coronário foi de 30,8 \pm 7,5 meses. Pacientes do grupo sem RLA tiveram porcentual significativamente mais alto de doença de um vaso $(82 \%$ vs. $47 \% ; \mathrm{P}<0,01)$. Pacientes do grupo sem RLA apresentaram taxa de ECAM de longo prazo de $21,7 \%$ (5 pacientes: 2 óbitos, 3 infartos do miocárdio), enquanto nos pacientes do grupo com RLA a taxa de ECAM foi de 9,8\% (8 pacientes: 1 óbito, 4 infartos do miocárdio, 3 cirurgias de revascularização miocárdica; $P=0,11)$. Conclusão: Pacientes com RIS clínica não tratados com RLA apresentaram doença de um vaso com maior frequência e tendência para maior incidência de ECAM em comparação com pacientes tratados com nova RLA.

DESCRITORES: Reestenose coronária. Angioplastia. Stents.

\section{ABSTRACT}

Clinical Outcomes of Patients with In-Stent Restenosis not Treated with a Revascularization Procedure

Background: Clinical restenosis after coronary stenting is generally treated by target vessel revascularization (TVR). This study was aimed at reporting the clinical profile and outcomes of patients with symptomatic in-stent restenosis (ISR) who were not submitted to a TVR. Method: Patients who presented clinical restenosis after coronary stenting between January 1997 and December 2001 were identified. Clinical and angiographic characteristics and clinical outcomes of patients who did not undergo a new revascularization (no-TVR group) were compared with revascularized patients (TVR group). All of the patients had at least 2 years of follow-up for the occurrence of major adverse cardiac events (MACE) after stent implantation. Results: In the study period, 1,221 stents were implanted in 1,149 patients. ISR was observed in 135 patients (12\%), of which 104 had complete clinical and angiographic follow-up, 23 in the no-TVR group and 81 in the TVR group. The mean follow-up period after coronary stenting was $30.8+7.5$ months. Patients in the no-TVR group had a significantly higher percent of one vessel disease $(82 \%$ vs. $47 \%$; $\mathrm{P}<0.01)$. Patients in the no-TVR group had a long-term MACE rate of $21.7 \%$ (5 patients: 2 deaths, 3 myocardial infarctions), whereas those submitted to a new revascularization showed a subsequent MACE rate of $9.8 \%$ (8 patients: 1 death, 4 myocardial infarctions, 3 coronary artery bypass graft surgery; $P=0.11$ ). Conclusion: Patients with clinical ISR not treated with a TVR more frequently presented one-vessel disease and a trend towards higher incidence of MACE when compared to those treated with a new TVR.

KEY-WORDS: Coronary restenosis. Angioplasty. Stents.

${ }^{1}$ Instituto de Cardiologia - Fundação Universitária de Cardiologia (IC-FUC) - Porto Alegre, RS, Brasil.

Correspondência: Alexandre Schaan de Quadros. Av. Princesa Isabel, 395 - Santana - Porto Alegre, RS, Brasil - CEP 90620-000 E-mail: alesq@terra.com.br

Recebido em: 18/9/2010 • Aceito em: 26/11/2010 
Gottschall CAM, et al. Resultados Clínicos de Pacientes com Reestenose Intrastent Não Tratada com Novo Procedimento de Revascularização. Rev Bras Cardiol Invasiva. 2010;18(4):419-23.

A reestenose é uma complicação relativamente comum da colocação do stent convencional e geralmente está associada a certas características clínicas e angiográficas, como diabetes melito, lesões longas e vasos de pequeno calibre. ${ }^{1-4}$ A reestenose clínica é definida pela ocorrência de angina e isquemia associada à recorrência de lesão intrastent ou dentro dos $5 \mathrm{~mm}$ das bordas do stent implantado. ${ }^{5}$ A apresentação clínica é geralmente uma angina progressiva e recomenda-se novo procedimento de revascularização para aliviar os sintomas. Recentemente demonstrou-se que um número considerável de pacientes com reestenose intrastent pode apresentar síndrome coronária aguda, com morbidade e mortalidade associadas. ${ }^{6-8}$ Entretanto, há escassez de dados relativos às consequências clínicas da reestenose intrastent não tratada. O objetivo deste estudo é relatar o perfil clínico e os desfechos de pacientes sintomáticos com reestenose intrastent grave que não foram tratados com outro procedimento de revascularização.

\section{MÉTODO}

\section{Pacientes}

Analisamos os dados relativos a todas as intervenções coronárias percutâneas com stents coronários como tratamento para doença coronária sintomática em nossa instituição entre janeiro de 1997 e dezembro de 2001. As características clínicas, do procedimento e angiográficas e os dados de acompanhamento foram registrados prospectivamente e incluídos em um banco de dados dedicado.

Foram identificados pacientes com reestenose clínica (lesão $\geq 50 \%$ em angiografia de controle associada a angina recorrente ou isquemia miocárdica em exames não-invasivos). Os grupos de estudo foram classificados de acordo com a realização ou não de nova revascularização da lesão-alvo para tratar a lesão reestenótica.

O estudo foi aprovado pelo comitê de ética da instituição. Os autores são os únicos responsáveis pelo desenho, condução e análise do estudo, elaboração e edição do texto e seu conteúdo final.

\section{Procedimento de implante}

Na ocasião do procedimento índex, todos os pacientes estavam recebendo aspirina por via oral (100$200 \mathrm{mg}$ diariamente) e ticlopidina na dose de $250 \mathrm{mg}$, duas vezes por dia. Foi recomendado o uso do tienopiridínico durante pelo menos um mês em todos os casos. As lesões foram tratadas usando técnicas padrão de intervenção coronária percutânea ${ }^{5}$ e as decisões técnicas específicas dos procedimentos ficaram a critério do operador.

A reintervenção em pacientes do grupo com revascularização da lesão-alvo consistiu em dilatação por balão, com relação balão-artéria de 1,1:1, visando à obtenção de estenose residual < 30\%. Um segundo stent foi implantado apenas para tratar dissecções residuais significativas. Aspirina e tienopiridínicos foram administrados novamente e mantidos durante pelo menos um mês.

\section{Análise angiográfica}

Todas as análises angiográficas foram realizadas em pelo menos duas projeções diferentes, por operadores experientes, com um sistema digital previamente validado (Siemens Axiom Artis, Munique, Alemanha), e nitroglicerina intracoronária foi administrada rotineiramente na dose de 100-200 $\mu \mathrm{g}$ antes das medições. O diâmetro do vaso-alvo foi definido como diâmetro médio dos segmentos luminais proximal e distal à lesão, e a gravidade da estenose foi avaliada em duas projeções ortogonais. O comprimento da lesão foi medido "de ponta a ponta" e as mais extensas foram consideradas uma única lesão apenas quando havia entre elas um segmento normal $<10 \mathrm{~mm}$ de comprimento.

\section{Acompanhamento tardio e desfechos do estudo}

Os pacientes foram acompanhados ambulatorialmente ou por contato telefônico. Em todos os casos, a avaliação angiográfica foi feita somente quando havia sintomas ou sinais de isquemia miocárdica recorrente. A decisão de realizar nova revascularização foi tomada de acordo com a opinião do médico responsável.

Todos os pacientes tiveram acompanhamento durante pelo menos dois anos após a colocação do stent. Após o diagnóstico da reestenose intrastent, os pacientes tiveram acompanhamento por mais um ano, quando foi verificada ocorrência de eventos cardíacos adversos maiores. Os eventos cardíacos adversos maiores foram definidos como óbito, infarto do miocárdio ou cirurgia de revascularização miocárdica. Infarto do miocárdio foi definido como creatina quinase fração $M B(C K-M B)>3$ vezes o normal, associado ou não ao desenvolvimento de novas ondas $\mathrm{Q}$ patológicas.

\section{Análise estatística}

As variáveis categóricas foram expressas como porcentual, e as variáveis contínuas foram expressas como média \pm desvio padrão. Todos os dados foram analisados por meio do software SPSS para Windows 11.0. As diferenças entre os grupos foram avaliadas pelo teste de qui-quadrado para as variáveis categóricas e pelo teste $t$ de Student para variáveis contínuas. Um valor de $\mathrm{P}<0,05$ foi considerado estatisticamente significante para todos os testes.

\section{RESULTADOS}

\section{Pacientes}

No período do estudo, 1.221 stents foram implantados em 1.149 pacientes. O primeiro acompanhamento foi concluído para $96 \%$ dos pacientes, com tempo 
Gottschall CAM, et al. Resultados Clínicos de Pacientes com Reestenose Intrastent Não Tratada com Novo Procedimento de Revascularização. Rev Bras Cardiol Invasiva. 2010;18(4):419-23.

médio de 13,4 \pm 5,2 meses após o implante do stent. Observou-se reestenose intrastent em 135 pacientes (12\%), dos quais 109 pacientes foram tratados com um novo procedimento de revascularização e 26 não realizaram um novo procedimento. Por não apresentarem dados angiográficos completos, 9 pacientes foram excluídos do estudo. Dos 126 pacientes restantes, 83\% tiveram segundo acompanhamento completo, com tempo médio de 17,5 \pm 11,2 meses após o diagnóstico angiográfico da reestenose. A população do estudo incluiu 104 pacientes, 23 não tratados com novos procedimentos de revascularização (grupo sem revascularização da lesão-alvo) e 81 pacientes revascularizados (grupo com revascularização da lesãoalvo). Os pacientes do grupo com revascularização da lesão-alvo foram tratados com nova intervenção coronária percutânea $(n=60)$ ou cirurgia de revascularização miocárdica $(n=21)$.

A Tabela 1 apresenta as características clínicas dos pacientes nos dois grupos de estudo. Não houve diferenças estatisticamente significantes para sexo, idade e fatores de risco para aterosclerose nos pacientes nãorevascularizados em comparação com aqueles submetidos a revascularização da lesão-alvo. O grupo com revascularização da lesão-alvo demonstrou maior prevalência de quadros coronários agudos, sem, no entanto, alcançar diferença estatística $(P=0,12)$.

A Tabela 2 apresenta as características angiográficas dos dois grupos. Os pacientes do grupo sem revascularização da lesão-alvo tiveram porcentagem significativamente maior de doença uniarterial em comparação àqueles do grupo com revascularização da lesão-alvo. A frequência de pacientes tratados com stents na artéria descendente anterior esquerda e de lesões ostiais e complexas foi semelhante entre os grupos. O diâmetro de referência do vaso e a extensão da lesão e do stent não apresentaram diferenças estatisticamente significantes, assim como o diâmetro luminal final. Os seguintes stents foram implantados: Multi-Link Tristar ${ }^{\mathrm{TM}}$ ou Tetra ${ }^{\mathrm{TM}}$ (Guidant Corp., Indianápolis, Estados Unidos), 40\%; Tenax ${ }^{\mathrm{TM}}$ (Biotronik, Berlim, Alemanha), 32\%; e BX Velocity ${ }^{\mathrm{TM}}$ (Cordis Corp., Miami, Estados Unidos), 28\%.

As características angiográficas das lesões reestenóticas são apresentadas na Tabela 3. A porcentagem de estenose foi semelhante nos grupos com e sem revascularização da lesão-alvo. A reestenose pré e pósstent também não foi estatisticamente diferente. O padrão de reestenose intrastent, focal ou difusa, também foi semelhante.

Em relação aos desfechos clínicos, observamos tendência a taxa de eventos cardíacos adversos maiores mais elevada nos pacientes com reestenose intrastent não tratados com novo procedimento de revascularização (21,7\%, 5 pacientes: 2 mortes, 3 infartos do miocárdio) em comparação aos que foram submetidos a revascularização $(9,8 \%, 8$ pacientes: 1 morte, 4 infartos do miocárdio, 3 enxertos da coronária) $(P=0,11)$.

\section{DISCUSSÃO}

Este foi um dos primeiros estudos a avaliar os desfechos clínicos de pacientes sintomáticos com reestenose intrastent comprovada por angiografia não tratados com um novo procedimento de revascularização. Nossos resultados demonstram que esses pacientes têm maior frequência de doença uniarterial e tendência a frequência menor de intervenção coronária percutânea prévia e quadros coronários agudos como apresentação clínica no procedimento índex. No acompanhamento clínico a longo prazo, os pacientes com reestenose intrastent não tratados com revascularização da lesão-alvo tiveram tendência a maior incidência de eventos cardíacos adversos maiores em comparação com aqueles que foram tratados com nova revascularização da lesão-alvo.

TABELA 1

Características clínicas

\begin{tabular}{lccc}
\hline & Sem RLA $(\mathbf{n}=\mathbf{2 3})$ & RLA $(\mathbf{n}=\mathbf{8 1})$ & P \\
\hline Sexo masculino, n (\%) & $14(59)$ & $62(76)$ & 0,18 \\
Idade, anos & $61 \pm 10,8$ & $58,3 \pm 11,7$ & 0,31 \\
Hipertensão, n (\%) & $16(68)$ & $49(60)$ & 0,47 \\
Dislipidemia, n (\%) & $11(47)$ & $33(41)$ & 0,63 \\
Diabetes melito, n (\%) & $7(32)$ & $31(38)$ & 0,62 \\
ICP prévia, n (\%) & $1(4)$ & $18(22)$ & 0,11 \\
Quadro clínico, n (\%) & $5(33)$ & $14(17)$ & 0,12 \\
Angina estável & $18(77)$ & $54(67)$ & $13(16)$ \\
Angina instável & 0 & 13 & \\
IM agudo & &
\end{tabular}

ICP = intervenção coronária percutânea; IM = infarto do miocárdio; $\mathrm{n}$ = número de pacientes; RLA = revascularização da lesão-alvo. 
Gottschall CAM, et al. Resultados Clínicos de Pacientes com Reestenose Intrastent Não Tratada com Novo Procedimento de Revascularização. Rev Bras Cardiol Invasiva. 2010;18(4):419-23.

TABELA 2

Características angiográficas e do procedimento

\begin{tabular}{lccc}
\hline & Sem RLA $(\mathbf{n}=\mathbf{2 3})$ & RLA (n= 81) & P \\
\hline Doença uniarterial, \% & $19(82)$ & $38(47)$ & $<0,01$ \\
DAE tratada, \% & $14(59)$ & $44(54)$ & 0,64 \\
Lesão ostial, \% & 0 & $6(7)$ & 0,33 \\
Lesão complexa, \% & $19(82)$ & $67(83)$ & $>0,99$ \\
Diâmetro do vaso de referência, mm & $3,05 \pm 0,34$ & $11 \pm 5,17 \pm 0,4$ & 0,19 \\
Extensão da lesão, mm & $10 \pm 4,1$ & $16,4 \pm 5,7$ & 0,42 \\
Comprimento do stent, mm & $15,2 \pm 4,9$ & $3,12 \pm 0,47$ & 0,36 \\
Diâmetro luminal final, mm & $3 \pm 0,37$ & 0,26 \\
\hline
\end{tabular}

DAE = descendente anterior esquerda; $\mathrm{n}$ = número de pacientes; RLA = revascularização da lesão-alvo.

TABELA 3

Características angiográficas das lesões reestenóticas

\begin{tabular}{lccc}
\hline & Sem RLA (n= 23) & RLA (n= 81) & P \\
\hline Estenose, \% & $74 \pm 19$ & $76 \pm 15$ & 0,6 \\
Reestenose pré-stent, n (\%) & $4(19)$ & $14(17)$ & $>0,99$ \\
Reestenose pós-stent, n (\%) & $6(25)$ & $11(13)$ & 0,2 \\
Padrão focal/difuso, n (\%) & $16(70)$ & $59(73)$ & 0,79 \\
\hline
\end{tabular}

n = número de pacientes; RLA = revascularização da lesão-alvo.

O impacto da reestenose na incidência de eventos cardíacos adversos maiores foi abordado pela primeira vez por Weintraub et al. ${ }^{9}$, ao compararem 1.570 pacientes com reestenose a 1.793 indivíduos sem reestenose. Após 6 anos de acompanhamento, a taxa de sobrevivência e de sobrevivência livre de infarto do miocárdio foi semelhante nos indivíduos com e sem reestenose e sua presença não foi associada a mortalidade na análise multivariada. Entretanto, quando apenas os pacientes com dor torácica recorrente foram analisados, os que tinham reestenose apresentaram taxas de eventos mais elevadas que aqueles sem reestenose. Esse achado é consistente com o relato atual, que incluiu apenas os pacientes com reestenose sintomática.

$\mathrm{Na}$ era dos stents, estudos recentes sugerem que a reestenose intrastent está associada a piores desfechos que a reestenose após angioplastia. Walters et al. ${ }^{8}$ compararam a apresentação clínica de 191 pacientes que desenvolveram reestenose intrastent com a de 71 pacientes com reestenose sem colocação de stent. As síndromes coronárias agudas (angina em repouso ou infarto agudo do miocárdio) foram mais frequentes nos pacientes com reestenose intrastent (68\% vs. $46 \% ; P=0,03)$, e esses pacientes tiveram maior probabilidade de ter trombos identificados por angiografia ( $9 \%$ vs. $0 \%$; $P=0,02)$. Concordante com esses achados, outros estudos também descreveram taxas elevadas de síndrome coronária aguda em pacientes com reestenose intrastent. ${ }^{6,7}$ Assali et al. ${ }^{10}$ também descreveram que uma apresentação de síndrome coronária aguda em pacientes com reestenose intrastent está associada a maior incidência de eventos cardiovasculares adversos recorrentes e reestenose angiográfica. Goldberg et al. ${ }^{11}$ demonstraram que alguns pacientes podem apresentar um padrão de reestenose intrastent agressiva, que ocorre mais cedo e é mais frequentemente associada a infarto do miocárdio.

Quando períodos de acompanhamento mais longos são considerados, vários estudos descreveram incidência muito baixa de eventos cardíacos adversos maiores relacionada à lesão-alvo após o primeiro ano, e a maioria dos eventos tardios foi atribuída à progressão da doença. ${ }^{12,13} \mathrm{Em}$ nosso estudo, apesar da incidência significativamente mais elevada de eventos cardíacos adversos maiores nos pacientes nãorevascularizados, devemos reconhecer que muitos desses pacientes continuaram livres de eventos cardíacos adversos maiores, pelo menos no período de acompanhamento descrito neste estudo. Esse é um achado intrigante e interessante, cujas razões não estão claras no presente estudo. Entretanto, sugerimos que estabilização ou regressão da estenose, remodelamento da coronária ou controle de sintomas com tratamento médico intenso poderiam ter tido um papel nesse caso. 
Gottschall CAM, et al. Resultados Clínicos de Pacientes com Reestenose Intrastent Não Tratada com Novo Procedimento de Revascularização. Rev Bras Cardiol Invasiva. 2010;18(4):419-23.

Alguns estudos demonstraram que lesões reestenóticas podem regredir ou continuar estáveis após o primeiro ano de implante do stent. Ormiston et al. ${ }^{14}$ demonstraram redução significativa da porcentagem de reestenose da lesão reestenótica em um estudo com 84 pacientes, principalmente naqueles com lesões mais graves. Hermiller et al. ${ }^{15}$ demonstraram achados semeIhantes em uma série independente com 23 pacientes. Nageh et al. ${ }^{16}$ também descreveram o caso de um paciente que se recusou a ser revascularizado em decorrência de uma reestenose intrastent e o acompanhamento angiográfico de dois anos revelou regressão significativa da lesão reestenótica. Entretanto, é importante enfatizar que apesar de a não recorrência de eventos cardíacos adversos maiores em vários pacientes com reestenose intrastent sintomática não-revascularizada ser um achado interessante e não esperado, não recomendamos adiar a revascularização nesses pacientes, já que as taxas de eventos cardíacos adversos maiores recorrentes são mais elevadas que naqueles que realizaram um novo procedimento de revascularização.

\section{Limitações do estudo}

Este estudo incluiu um pequeno número de pacientes, principalmente porque a reestenose intrastent clínica não tratada com um novo procedimento de revascularização é uma situação rara. Por essa razão, o pequeno número de desfechos impediu a realização de uma análise multivariada para ajustar os fatores de confusão entre os dois grupos de estudo. Não houve acompanhamento clínico da condição da angina nem informações sobre os medicamentos utilizados nos dois grupos de pacientes. É possível que a recusa para realizar um novo procedimento de revascularização esteja associada à não aderência aos medicamentos prescritos e à falta de acompanhamento clínico, fortes preditores de piores resultados clínicos. Também não temos informações sobre a viabilidade do miocárdio dos pacientes tratados/não tratados, importante fator prognóstico para desfechos clínicos a longo prazo. As taxas de acompanhamento completo na segunda entrevista dos pacientes foram mais baixas, mas uma comparação entre as características clínicas daqueles com e sem acompanhamento não revelaram diferenças significativas.

\section{CONCLUSÃO}

Os pacientes com reestenose clínica não tratados com um novo procedimento de revascularização tiveram tendência a menor frequência de intervenção coronária percutânea prévia e quadros coronários agudos, como apresentação clínica no procedimento índex, e demonstraram maior frequência de doença uniarterial. No acompanhamento clínico a longo prazo, as taxas de eventos cardíacos adversos maiores foram duas vezes maiores nesses pacientes em comparação com os que foram revascularizados, com um valor de $\mathrm{P}$ com significância estatística limítrofe.

\section{CONFLITO DE INTERESSES}

Os autores declararam inexistência de conflito de interesses relacionado a este manuscrito.

\section{REFERÊNCIAS}

1. Elezi S, Kastrati A, Neumann FJ, Hadamitzky M, Dirschinger J, Schömig A. Vessel size and long-term outcome after coronary stent placement. Circulation. 1998;98(18):1875-80.

2. Bauters C, Hubert E, Prat A, Bougrimi K, Van Belle E, McFadden $E P$, et al. Predictors of restenosis after coronary stent implantation. J Am Coll Cardiol. 1998;31(6):1291-8.

3. Abizaid A, Kornowski R, Mintz GS, Hong MK, Abizaid AS, Mehran $R$, et al. The influence of diabetes mellitus on acute and late clinical outcomes following coronary stent implantation. J Am Coll Cardiol. 1998;32(3):584-9.

4. Gottschall CA, Quadros AS, Sarmento-Leite R. Predictive score for target vessel revascularization after bare metal coronary stenting. J Invasive Cardiol. 2006;18(1):22-6.

5. Smith SC Jr, Feldman TE, Hirshfeld JW Jr, Jacobs AK, Kern MJ, King SB $3^{\text {rd }}$, et al. ACC/AHA/SCAI 2005 Guideline Update for Percutaneous Coronary Intervention - summary article: a report of the American College of Cardiology/American Heart Association Task Force on Practice Guidelines (ACC/AHA/SCAI Writing Committee to Update the 2001 Guidelines for Percutaneous Coronary Intervention). Circulation. 2006;113(7):156-75.

6. Chen MS, John JM, Chew DP, Lee DS, Ellis SG, Bhatt DL. Bare metal stent restenosis is not a benign clinical entity. Am Heart J. 2006;151(6):1260-4.

7. Nayak AK, Kawamura A, Nesto RW, Davis G, Jarbeau J, Pyne $\mathrm{CT}$, et al. Myocardial infarction as a presentation of clinical in-stent restenosis. Circ J. 2006;70(8):1026-9.

8. Walters DL, Harding SA, Walsh CR, Wong P, Pomerantsev E, Jang IK. Acute coronary syndrome is a common clinical presentation of in-stent restenosis. Am J Cardiol. 2002;89(5):491-4.

9. Weintraub WS, Ghazzal ZMB, Douglas JS Jr, Liberman HA, Morris DC, Cohen CL, et al. Long-term clinical follow-up in patients with angiographic restudy after successful angioplasty. Circulation. 1993;87(3):831-40.

10. Assali AR, Moustapha A, Sdringola S, Denktas AE, Willerson JT, Holmes DR Jr, et al. Acute coronary syndrome may occur with in-stent restenosis and is associated with adverse outcomes (the PRESTO trial). Am J Cardiol. 2006;98(6):729-33.

11. Goldberg SL, Loussararian A, De Gregorio J, Di Mario C, Albiero R, Colombo A. Predictors of diffuse and aggressive intra-stent restenosis. J Am Coll Cardiol. 2001;37(4):1019-25.

12. Cutlip DE, Chauhan MS, Baim DS, Ho KK, Popma JJ, Carrozza $J P$, et al. Clinical restenosis after coronary stenting: perspectives from multicenter clinical trials. J Am Coll Cardiol. 2002; 40(12):2082-9.

13. Kimura T, Abe K, Shizuta S, Odashiro K, Yoshida Y, Sakai $K$, et al. Long-term clinical and angiographic follow-up after coronary stent placement in native coronary arteries. Circulation. 2002;105(25):2986-91.

14. Ormiston JA, Stewart FM, Roche AH, Webber BJ, Whitlock RM, Webster MW. Late regression of the dilated site after coronary angioplasty: a 5-year quantitative angiographic study. Circulation. 1997;96(2):468-74.

15. Hermiller JB, Fry ET, Peters TF, Orr CM, Van Tassel J, Waller $\mathrm{B}$, et al. Late coronary artery stenosis regression within the Gianturco-Roubin intracoronary stent. Am J Cardiol. 1996; $77(4): 247-51$.

16. Nageh T, Duncan E, Thomas MR. Regression of coronary instent restenosis. Catheter Cardiovasc Interv. 2004;62(2):201-2. 